\title{
Universal Battery Performance and Degradation Model for Electric Aircraft
}

Alexander Bills ${ }^{1}$, Shashank Sripad ${ }^{1}$, William L. Fredericks ${ }^{1}$, Matthew Guttenberg ${ }^{1}$, Devin Charles $^{2}$, Evan Frank $^{2}$, Venkatasubramanian Viswanathan ${ }^{1,3}$

${ }^{1}$ Department of Mechanical Engineering, Carnegie Mellon University, Pittsburgh, Pennsylvania, 15213, USA.

${ }^{2} A^{\wedge} 3$ by Airbus, Santa Clara, California, 95050, USA.

${ }^{3}$ Wilton E. Scott Institute for Energy Innovation, Carnegie Mellon University, Pittsburgh, Pennsylvania, 15213, USA. 
Electric mobility application design requires accurate and fast prediction of Li-ion battery performance and life. An emerging area of mobility enabled by electrification is electric vertical takeoff and landing aircraft (eVTOL) for urban air mobility (UAM). Design process, techno-economic analysis and the operation of on-board systems depend on the fast and accurate prediction of Li-ion battery performance and life. These models must be accurate for the unique use-case of UAM requiring high power at takeoff and landing, thereby, causing significant rise in battery cell temperature. In this work, we generate a battery performance and thermal dataset specific to eVTOL use-cases and develop a fast and accurate performance and degradation model around that dataset. We use a machine-learning based physicsinformed battery performance model to break the typically observed accuracy-computing cost trade-off. We fit the aging parameters for each cycle in a given cell's lifetime, and then model the evolution of those parameters using a new approach that combines traditional physics-based models, consisting of SEI film growth, charge loss, and Li Plating, along with a neural network in a universal ordinary differential equations (u-ODEs) framework. The new approach provides a unique way to combine the extrapolative capability of physics-based models with the representation power of neural networks.

Electric vehicles are seeing an increased mass-market adoption due to the rapidly falling cost and increasing performance of Li-ion batteries. ${ }^{1+\frac{3}{3}}$ These improvements are enabling the electrification of aviation powertrains with many demonstration flights for short distances. ${ }^{4 / 5}$ In an electric aircraft, power is transferred from the battery energy storage system to propulsive thrust by a high voltage distribution bus, electric motor, and power inverter. Relative to internal com- 
bustion or turbine engine systems, this mode of propulsive energy transfer facilitates elimination of the complexity associated with gearboxes or mechanical transmissions, and affords relatively low unit costs per propulsor (inverter, motor, propeller) ${ }^{6}$ This paradigm shift in aircraft propulsion system has enabled a vast array of new hybrid and electric aircraft configurations. ${ }^{7}$ Many electric aircraft designs utilize distributed electric propulsion to realize novel configurations which can achieve a significant safety and efficiency advantage over conventional single or multi-engine aircraft. ${ }^{[8}$ Notably, these developments in electrical energy storage and distributed electric propulsion have enabled the possibility of urban air mobility (UAM). As outlined by NASA, UAM aims to safely and efficiently transport passengers and cargo in an urban area,, 9 and may have an energyefficiency 10 advantage over ground transport. Core to the UAM concept are vertical take-off and landing aircraft (eVTOLs), that use rotors to take off and land without needing a runway. 11

The design trade-offs using Li-ion based electric aircraft designs are distinct from those in combustion engines, in large part due to orders of magnitude difference in specific energy between Li-ion batteries and jet-fuel. Thus, electric aircraft require careful integration and use of Li-ion battery systems and battery weight directly limits the electric aircraft range ${ }^{11}{ }^{12}$ Most eVTOL aircraft are typically designed for the critical case: cruise to maximum range into a headwind, followed by a redirect reserve segment and a subsequent contingency landing such as a single propulsor failure. This mission profile is especially challenging to achieve at the battery retirement stateof-health (typically $80-85 \% \mathrm{SOH}$ ), as maximum power output is demanded at minimum state-ofcharge (SOC) ${ }^{11}$ To ensure that the co-design of electric propulsion sub-systems is consistent with the sized vehicle geometry and weights, a rapid battery performance estimation method is required 
in the sizing loop ${ }^{[13}$ In addition, on-board state-estimation of energy and power is important for aircraft operations. Battery $\mathrm{SOH}$ estimation as a function of mission profile is also critical for techno-economic and business case analyses, as batteries are a major component of operations $\operatorname{cost} .14$

Estimating the state-of-charge over a duty cycle and the state-of-health over the lifetime of a cell has been performed using one of three broad categories of models, (i) empirical models, (ii) physics-based models, $\frac{[15}{,}$ or (iii) data-driven methods $!^{16}$ Each category of models entails a number of advantages and disadvantages based on the accuracy, the computational time to run the model, the interpretability of the model results and extrapolation capability of the models. A typical trade-off plot between model accuracy and computational time is shown in Fig. 1a! ${ }^{16}$ The ability to break this kind of multi-scale modeling trade-off by physics-informed neural networks is now widely realized in atomistic simulations ${ }^{18}$ and fluid mechanics ${ }^{19}$ The key insight that enables breaking this trade-off is to encode physical principles for data efficiency and extrapolation in conjunction with the representation power of neural networks. In this work, we develop such an approach, CellFit, a physics-constrained machine-learning model for battery performance, thermal response, and degradation. A simplified physical representation that obeys conservation laws (charge, energy) are used together with the representation power of neural networks towards a class of models which we term as universal battery performance and degradation models. The universality arises from describing the residual mechanisms, not captured by physical models, by the representation power of neural networks. The model is open-source and built using the Julia programming language, $\underset{20}{,}$ enabling great computational speed of less than $1 \mathrm{~ms}$ for a cycle. 
a.

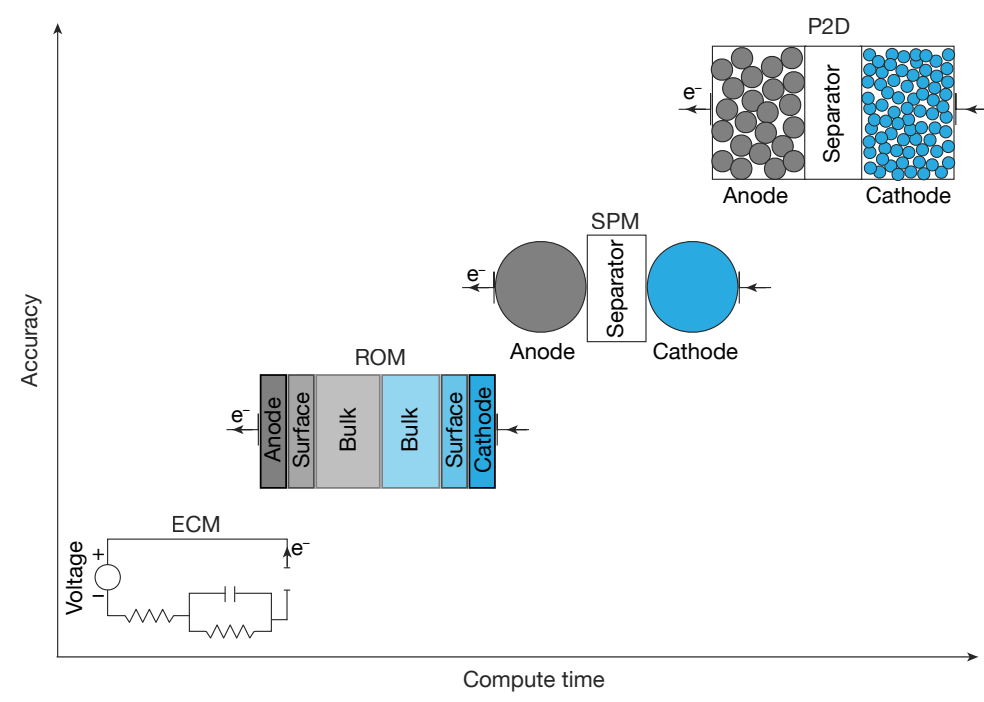

b.
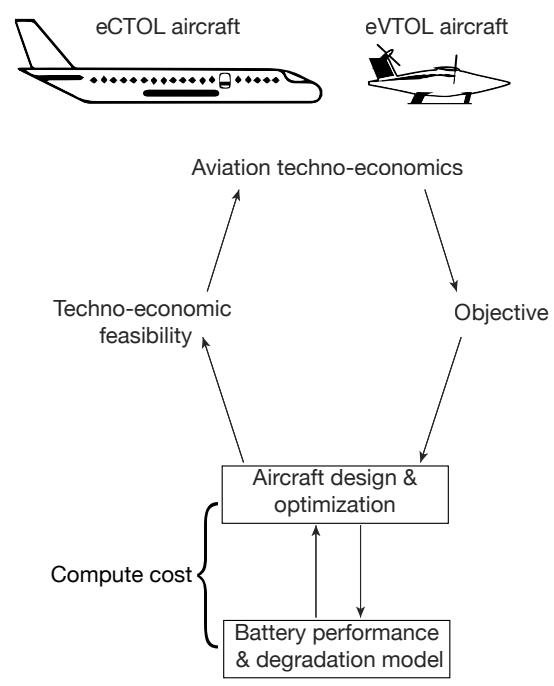

Figure 1: Model accuracy v. computational time and its importance in electric aviation (a)

Model accuracy and computational time trade-off shown for different battery performance models. Electrochemical models such as Pseudo 2 dimensional (P2D) models and single particle models (SPM) are typically accurate and interpretable, but parameter estimation is difficult and model runtime is long. Equivalent circuit models (ECM) provide fast models at the cost of accuracy and interpretability. We propose that reduced order models supplemented with data-driven and machine learning approaches provide an accurate and fast compromise which provides some level of physical interpretability as the optimal battery model for electric aircraft development and design. (b) Aircraft require careful integration of battery modeling to ensure safety, particularly in the early stages of design and development. The model must be able to account for the full life cycle of the battery by incorporating a degradation model to ensure safety and to estimate economic viability. Additionally, the battery model must have a fast runtime because of the iterative nature and compuatational complexity of aircraft design 
The development of machine learning based models requires relevant battery performance datasets to train and test the models. While datasets exist for testing Li-ion performance under a few duty cycles, ${ }^{2|17| 21}$ the limited number of openly-available datasets remains a challenge to bring advanced machine-learning methods to battery performance prediction, $\frac{17 / 22}{12}$ and there are no such datasets specific to electric aircraft missions. To fill this gap, we generate an experimental battery performance dataset using cell testing specific to the operating conditions encountered by an eVTOL. The mission profiles follow the same generic format in all cases: (i) Take-off: the cell was discharged at constant power at a high rate for a period, $t_{t o}$ (ii) Cruise: the cell was discharged at constant power at a lower rate for a longer duration, $t_{c r}$ and (iii) Landing: the cell was discharged at constant power at the same rate as takeoff for a slightly longer period of time, $t_{l a}$, (iv) Rest: the cell was allowed to rest until it cooled to a temperature of less than $27^{\circ} \mathrm{C}$, (v) Charging: the cell was charged using a constant current-constant voltage (CC-CV) charging protocol, (vi) Rest: The cell was allowed to rest until cell temperature reached $35^{\circ} \mathrm{C}$, then allowed to rest 15 minutes further before beginning the next cycle. Every 50 cycles, the cells underwent a capacity test wherein they were discharged at constant current until their voltage was less than $2.5 \mathrm{~V}$. The cells were cycled until they reached less than $2.5 \mathrm{~V}$ or a temperature of greater than $70^{\circ} \mathrm{C}$ during a typical discharge cycle. The dataset was carefully curated to represent a diverse set of use-cases to test the generalizability of the developed model.

For performance modeling, we use a physics inspired model ${ }^{23}$ that obeys conservation laws and take a data-driven approach to estimate the parameters. Parameter estimation for the model is performed against the experimentally generated eVTOL battery performance dataset. Battery 
performance dataset typically contains time-series data of voltage, current, and temperature. Parameter estimation for electrochemical battery models is a notable challenge $\mathrm{e}^{24}$. We use simulated annealing ${ }^{25}$ to estimate the model parameters required for predicting the voltage of the cell over a given duty cycle. Parameters used in this model cannot be directly obtained from physical measurements. However, they are physically motivated and correspond to processes which include electrochemical reactions, reaction kinetics, and transport. The performance model is a system of ordinary differential equations which obeys physical constraints, including conservation of charge, conservation of energy, the second law of thermodynamics for open-circuit voltage, and kinetic rate-laws of electrochemical reactions, as further described in the methods section. This combination strikes a compromise between accuracy, computational speed, and interpretability of the model.

The operation of on-board electric propulsion system requires fast and accurate prediction of battery performance in terms the cell terminal voltage and the cell temperature over the mission. The cell voltage and temperature prediction from CellFit for a wide range of experimentally tested mission profiles is shown in Figure 2. Two missions are shown in Figure 2, at two different points in the cycle life. The first is VAH01, the baseline mission. The second is VAH06, which has a relatively low charging current. For VAH01, we show the discharge curve at the first and the $800^{\text {th }}$ cycle, and for VAH06 we show the discharge curve at the first and the $701^{\text {st }}$ cycle. Overall, the model is extremely accurate. Particularly important for the application of eVTOL design, the model accurately predicts the highest temperature of the cell. Much of the thermal error results when the cell is cooling during the first rest period. This error can be attributed to the varying 
cooling conditions (convective cooling coefficient) experienced by the cell during the experiments. In all cases, the model predicts a slightly lower voltage during discharge and a slightly higher voltage during charge than the experimental results. This may be indicative of the model having a slightly higher resistance in an error to accurately capture the highest temperature.

The discharge curves shown in Figure $2 \mathrm{a}$ and $\mathrm{b}$ are important to model throughout the life of the aircraft to inform design decisions based on battery performance characteristics. Figure $2 \mathrm{k}$ also shows the loss function which defines the accuracy of performance model for each cycle over the lifetime of the battery. We used a tuned loss function to emphasize the importance of the prediction of the quantities of interest (the details of which can be found in the methods section). Figure 2 also shows the value of the loss function over cycle life for all of the cycles. The loss does not substantially increase with time, and for most cycles, it stays relatively low. For reference, the loss functions for the baseline mission and for the VAH06 mission shown in Figure $2 \mathrm{a}$ and $\mathrm{b}$ are around 0.2 and 0.15 respectively. Therefore, the error in predicting quantities of interest are consistently low throughout the lifecycle of the battery. Additionally, each discharge profile takes less than 1 millisecond to generate. Therefore due to its accuracy over the battery's lifetime and the overall model speed, this framework and model is ideal for design of eVTOL's.

Each cycle of a mission, as represented by each point shown in Figure 2 e, is defined by a unique set of parameter values to predict the voltage and temperature over the cycle. We refer to this set of parameter values as performance parameters. A subset of the performance parameters is chosen to capture the evolution of the performance model as the battery ages ${ }^{26}$, we refer to this 

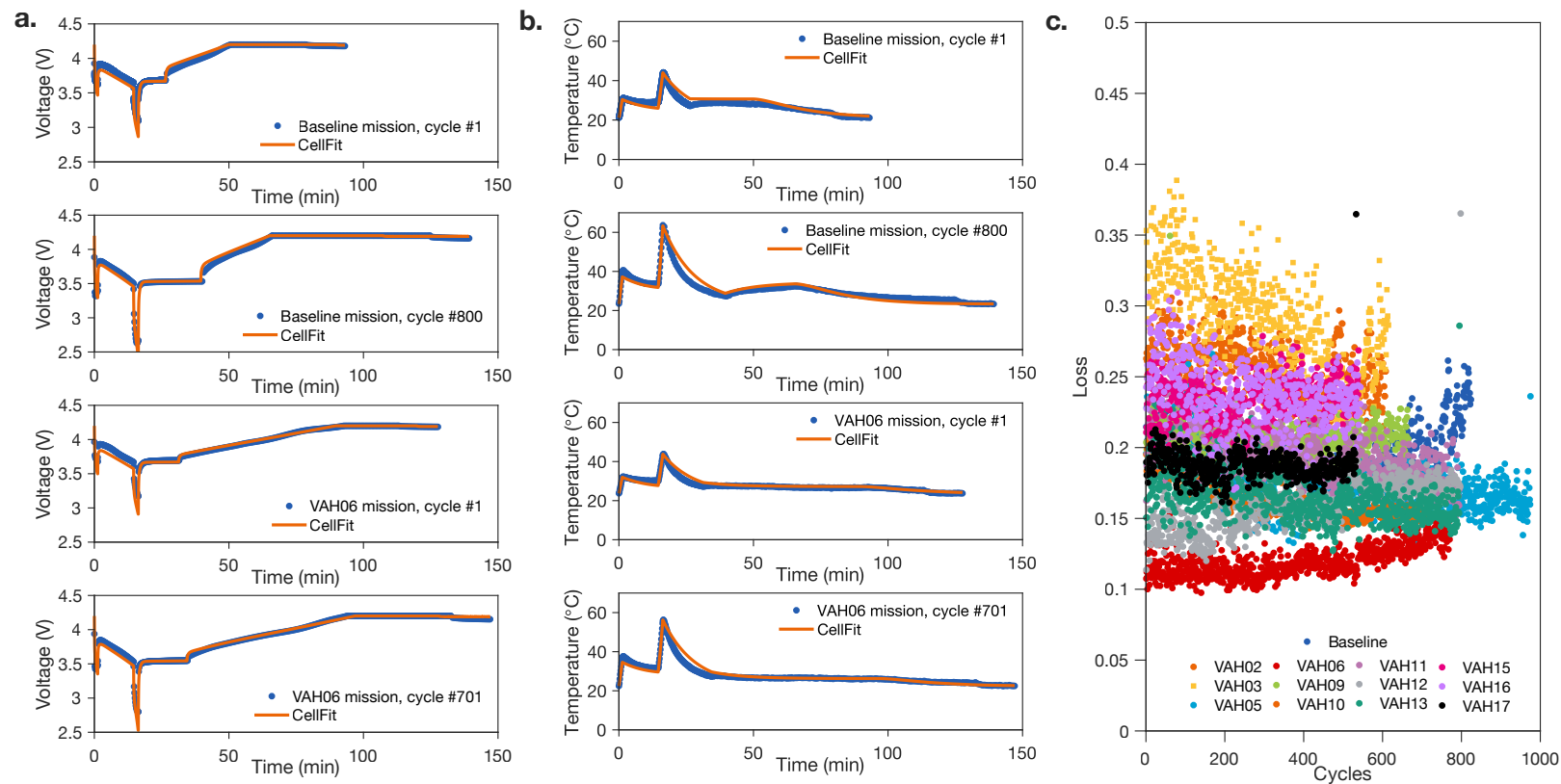

Figure 2: CellFit voltage and temperature predictions and its accuracy Voltage and temperature predictions generated at a sub-millisecond run time of CellFit(a) for the Baseline mission (VAH01) at cycle 1 and 800, (b) for the VAH06 mission (which has a lower charge rate than the baseline condition) at cycle 1 and 701. Importantly for safety and thermal management purposes in the aircraft design process, the temperature prediction is nearly always accurate for the highest temperature experienced during any given cycle. (c) Numerical value of the dimensionless loss function for temperature and voltage predictions. While some missions are better than others, and some issues exist at the battery's end of life most remain relatively low and flat throughout the lifecycle of the battery. 
subset as the aging parameters. Additionally, to capture the evolution of the aging parameters over the lifetime of the battery, we implement a model to evolve the aging parameters, and we refer to the parameters of that model as the degradation parameters.

The smallest set of aging parameters must be first be identified to model degradation in this framework. This, in a process akin to hyperparameter optimization, is accomplished by first picking a set of initial parameters (typically based on physical intuition), then calculating the loss function for each cycle in the dataset, as shown in Figure 2r. To calculate the loss, the aging parameters must be estimated, and the parameter estimation routine for these parameters is slightly different than for the other parameters, as detailed in methods. The loss function for each cycle over the life of the battery should be low and flat, indicating that the model, with estimated aging parameters and constant non-aging performance parameters, is capable of successfully predicting battery aging. An objective function, e.g. deviation from initial loss, could be used to evaluate whether the parameter set is sufficient to capture the variations. Once the smallest set of parameters which meets the above loss criteria has been identified, a degradation model must be chosen and fit for the evolution of those parameters.

There are several advantages to the degradation modeling approach followed here. Firstly, the parameter estimation process for each cycle over the lifetime of the battery (the aging parameters) can be conducted independently, therefore, can be conducted in parallel, utilizing modern high performance computing architectures such as multi-core architectures and graphics processing units. Estimating the parameters in parallel theoretically allows us to estimate the parameters 
for each cycle over entire lifetime of a battery in the time required for a single cycle. Secondly, this approach results in accurate prediction of discharge characteristics throughout the battery's lifetime, which is vital for design, analysis, and safe operation of electric aircraft.

This degradation framework allows for use of both physics-based and machine-learning based degradation models. We developed two degradation models: (i) A physics-based mechanistic degradation model (MDM), which includes contributions from three different degradation mechanisms: SEI growth, lithium plating, and active material loss. ${ }^{[15 / 27 / 28}$ (ii) A novel approach, called the universal battery degradation model (UBDM), which is based on neural differential equations and universal ordinary differential equations ${ }^{29 / 30}$. In the UBDM approach, we use a neural network to supplement the mechanistic model to capture complex effects of charge and active material loss as well as change in resistance. Any mechanistic model can be substituted for our mechanistic model in the UBDM, to account for changes in chemistry, operating conditions, cell models, etc.

The dataset is split into a training set and a testing set to train both degradation models (see Methods for details). The datasets are selected to reflect the broad nature of the generated dataset, attempting to keep experiments/ missions of each kind in both the testing set and the training set. MDM and UBDM are then trained alongside each other. Figure 3r shows the total error experienced by training and test. For nearly all sets, the UBDM model performs better than the MDM model. For the training set, the UBDM experiences a total accuracy increase of around 15\%. For the testing set, the UBDM experiences a total accuracy increase of around 19\%. This 
a.
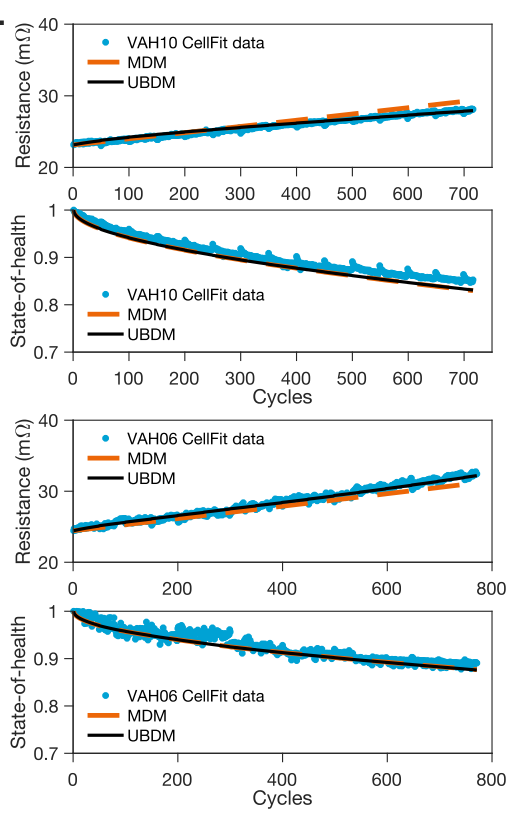

b.
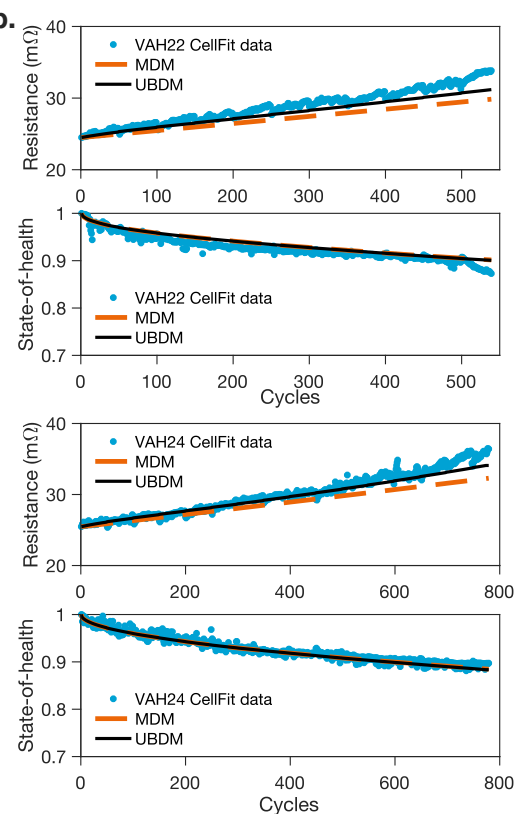

c. 3
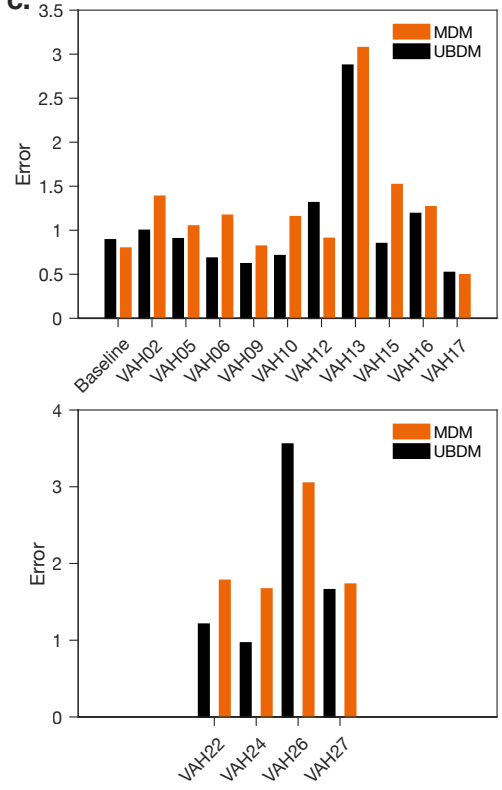

Figure 3: Comparison of UBDM and Mechanistic Degradation Model (a) Resistance and Charge for the MDM and the UBDM for two missions in the training set. The UBDM has learned when resistance should follow a sublinear growth pattern and when it should follow a superlinear growth pattern depending on the data. (b) Resistance and Charge for the MDM and the UBDM for two missions in the testing set. The UBDM outperforms the MDM in all cases, showing its generalization ability. (c) Errors for the MDM and the UBDM for the training set and the testing set. In nearly all training cases and in all testing cases, the UBDM outperforms the MDM. 
represents a substantial improvement for an already relatively accurate model. The MDM shows a linear increase in resistance over the life of the cell. The UBDM corrects this model by slowing growth in some cases, and speeding growth in some cases, as shown in Figure $3 \mathrm{a}$ and Figure $3 \mathrm{~b}$ for the testing set. These differences show that the model is not overfitting to anomalies in the dataset, but rather is learning the underlying patterns of degradation.

The ability to augment physics-constrained features with neural networks is causing a revolution in multi-scale modeling in various fields. $\frac{1819}{19}$ We believe that the ideas behind the universal battery modeling approach presented here will have a similar impact in modeling across length and time scales, capturing microscopic and macroscopic phenomena at unprecedented detail.

\section{Conclusions}

In this work, we have generated a battery performance and thermal response dataset specific to the urban air mobility use-case. We expect this dataset to rapidly accelerate the nascent field of batteries for electric aircraft. In order to learn this dataset, we develop a 'universal' battery modeling approach, which combines exact physical laws along with neural networks that can learn the residual (missing) physics. Using this modeling approach, we show incredible accuracy and computational efficiency for voltage, temperature and degradation for the dataset. The universal modeling framework developed here has the potential to disrupt battery modeling akin to similar disruptions occurring in quantum mechanics of materials, fluid mechanics by physics-constrained neural networks. 


\begin{tabular}{||lcccc||}
\hline Phase & Duration & Power & C-rate & Discharge Energy \\
\hline \hline Take-off & $75 \mathrm{~s}$ & $54 \mathrm{~W}$ & $5 \mathrm{C}$ & $1.12 \mathrm{Wh}$ \\
\hline Cruise & $800 \mathrm{~s}$ & $16 \mathrm{~W}$ & $1.48 \mathrm{C}$ & $3.55 \mathrm{Wh}$ \\
\hline Landing & $105 \mathrm{~s}$ & $54 \mathrm{~W}$ & $5 \mathrm{C}$ & $1.57 \mathrm{Wh}$ \\
\hline
\end{tabular}

Table 1: Baseline Mission Parameters (Discharge)

\section{Methods}

Cell Testing and Data Generation: This work utilized Sony-Murata 18650 VTC-6 cells (INR technology). This cell has a rated capacity of $3000 \mathrm{mAh}$ at a nominal voltage of $3.6 \mathrm{~V}$. The manufacturer specified maximum continuous discharge rate is $10 \mathrm{C}$ with a $80^{\circ} \mathrm{C}$ upper temperature cut off. This cell is appropriate for evaluation in eVTOL applications as it can sustain high power demand while providing a cell specific energy of $230 \mathrm{Wh} / \mathrm{kg}$.

All cells were tested in a Arbin 200A cylindrical cell holder paired with a BioLogic BCS815 modular battery cycler. As shown in Supplementary Figure 1, cell testing was performed in enclosures that were placed in a temperature chamber that was maintained at $25^{\circ} \mathrm{C}$. Cell can surface temperatures were measured via a thermocouple fixed to the cell body with aluminum tape.

Generation of the eVTOL data set was developed from the baseline power profile for a notional eVTOL flight given in table 1 .

Battery cell state-of-health was measured for the baseline mission profile and a series of 


\begin{tabular}{||ccc||}
\hline Phase & Definition & End Criteria \\
\hline Rest 1 & 0 Amps & $T<27^{\circ} \mathrm{C}$ \\
\hline CC Charge & $1 \mathrm{C}$ & $V>4.2$ \\
\hline CV Charge & $\mathrm{V}=4.2$ & $I<C / 30$ \\
\hline Rest 2 & $\mathrm{I}=0$ & $T<35^{\circ} \mathrm{C}$ \\
\hline
\end{tabular}

Table 2: Baseline Mission Parameters (Charge)

variations on the baseline mission. For each mission profile, cells were cycled until cell voltage reached $2.5 \mathrm{~V}$ or cell temperature reached $70 \mathrm{C}$ during discharge. To measure cell energy capacity a specific reference test was performed at the start of each aging test campaign and following each subsequent set of 50 mission cycles. Capacity was measured by discharging the cell from $100 \%$ to $0 \%$ state-of-charge at a discharge rate of $\mathrm{C} / 5$. These reference tests also included internal resistance measurement at 20 percent and 60 percent depth of discharge. After all discharge cycles a rest time was observed to allow the cell to cool to $30^{\circ} \mathrm{C}$. Once $30^{\circ} \mathrm{C}$ cell temperature was reached a CC-CV charge profile was started with constant current charge at $1 \mathrm{C}$, followed by constant voltage charge until charge current decayed to $\mathrm{C} / 30$ to a (nominally) $4.2 \mathrm{~V}$ end of charge voltage.

To evaluate the impact of several test parameters on cell aging, a single variable in the baseline mission profile was modified according to the test descriptions given in Table 3 and Table 4

Every 50 cycles, the cells underwent a capacity test, wherein they were discharged at 0.6 amps until their voltage dropped below $2.5 \mathrm{~V}$. The tests were repeated until the cells dropped below 
$2.5 \mathrm{~V}$ or the temperature rose above $70^{\circ} \mathrm{C}$ during a mission cycle.

Cell Model: The cell model is based on an adaptation of the model presented by Daigle and Kulkarni ${ }^{31}$. It is a fully-coupled electrochemical-thermal model which, while being inspired by more accurate battery models such as the Newman model, represents a significant simplification of those models. It reduces the system of partial differential equations typically used to model electrochemical reactions to a system of ordinary differential equations to perform fast integration. It models only the cathode and anode of the system, neglecting all electrolyte dynamics. It has two principal components: Modeling charge (q) movement and potential (V). The model comprises 4 domains: bulk and surface regions of both the cathode and the anode. Charge flow is governed by applied current between the surfaces of each electrode and by diffusion between the surface and bulk of each electrode. Voltage is calculated using a buildup consisting of 5 terms: cathode and anode equilibrium potentials, cathodic and anodic surface overpotentials (modeled by the butler volmer equations), and an ohmic overpotential. All overpotentials are also passed through first-order filters to prevent sudden voltage changes. Cell resistance is modeled by a lumped resistance parameter. The anode and cathode equilibrium potentials are modeled by the Redlich-Kister polynomials.

The thermal model is based on a lumped parameter model with heat generation terms for ohmic heating and entropic heating. Cooling is convective cooling, owing to the test setup as described above.

The equations for the charge transport portion of the model are given in equations (1), where 


\begin{tabular}{||c|l||}
\hline \multicolumn{2}{|c||}{ Training Data } \\
\hline Mission Profile & Description \\
\hline VAH01 & Baseline mission \\
VAH02 & Cruise time, $t_{c}=1000 \mathrm{~s}$ \\
VAH05 & Power reduction of 10 percent for takeoff, cruise, and landing \\
VAH06 & CC-CV charge cycle at $\mathrm{C} / 2 \mathrm{CC}-\mathrm{C} / 30 \mathrm{CV}$ \\
VAH09 & Thermal chamber temperature of $20^{\circ} \mathrm{C}$ \\
VAH10 & Thermal chamber temperature of $30^{\circ} \mathrm{C}$ \\
VAH11 & Power reduction of $20 \%$ for takeoff, cruise, and landing \\
VAH12 & Cruise time, $t_{c}=400 \mathrm{~s}$ \\
VAH13 & Cruise time, $t_{c}=600 \mathrm{~s}$ \\
VAH15 & Cruise time, $t_{c}=1000 \mathrm{~s}$ \\
VAH16 & CC-CV charge cycle at $1.5 \mathrm{C}$ CC $-\mathrm{C} / 30 \mathrm{CV}$ \\
VAH17 & Baseline mission \\
\hline
\end{tabular}

Table 3: Descriptions of the Training dataset 


\begin{tabular}{||c|l||}
\hline \multicolumn{2}{|c||}{ Testing and Validation Data } \\
\hline Mission Profile & Description \\
\hline VAH07 & End of charge voltage at 4.0V \\
VAH20 & CC-CV charge cycle at $1.5 \mathrm{C} \mathrm{CC}-\mathrm{C} / 30 \mathrm{CV}$ \\
VAH22 & Cruise time of 1000s \\
VAH23 & End of charge voltage at 4.1V \\
VAH24 & CC-CV charge cycle at C/2 CC - C/30 CV \\
VAH25 & Thermal chamber temperature of 20C \\
VAH26 & Cruise time of 600s \\
VAH27 & Baseline \\
VAH28 & Power reduction of 10 percent for takeoff, cruise, and landing \\
VAH30 & Thermal chamber temperature of 35 ${ }^{\circ} \mathrm{C}$ \\
\hline
\end{tabular}

Table 4: Descriptions of the Testing and Validation dataset 
$q$ represents the amount of charge in a domain, $v$ represents the capacity of that domain, and $D$ represents the diffusion coefficient. The subscripts $s$ and $b$ indicate surface and bulk respectively, and the subscripts $p$ and $n$ indicate positive and negative respectively.

$$
\begin{gathered}
\frac{\mathrm{dq}_{\mathrm{sp}}}{\mathrm{dt}}=\frac{1}{\mathrm{D}}\left(\frac{\mathrm{q}_{\mathrm{bp}}}{\mathrm{v}_{\mathrm{bp}}}-\frac{\mathrm{q}_{\mathrm{sp}}}{\mathrm{v}_{\mathrm{sp}}}\right)+\mathrm{I}_{\mathrm{app}} \\
\frac{\mathrm{dq}_{\mathrm{bp}}}{\mathrm{dt}}=-\frac{1}{\mathrm{D}}\left(\frac{\mathrm{q}_{\mathrm{bp}}}{\mathrm{v}_{\mathrm{bp}}}-\frac{\mathrm{q}_{\mathrm{sp}}}{\mathrm{v}_{\mathrm{sp}}}\right) \\
\frac{\mathrm{dq}_{\mathrm{sn}}}{\mathrm{dt}}=\frac{1}{\mathrm{D}}\left(\frac{\mathrm{q}_{\mathrm{bn}}}{\mathrm{v}_{\mathrm{bn}}}-\frac{\mathrm{q}_{\mathrm{sn}}}{\mathrm{v}_{\mathrm{sn}}}\right)-\mathrm{I}_{\mathrm{app}} \\
\frac{\mathrm{dq} \mathrm{q}_{\mathrm{bn}}}{\mathrm{dt}}=-\frac{1}{\mathrm{D}}\left(\frac{\mathrm{q}_{\mathrm{bn}}}{\mathrm{v}_{\mathrm{bn}}}-\frac{\mathrm{q}_{\mathrm{sn}}}{\mathrm{v}_{\mathrm{sn}}}\right)
\end{gathered}
$$

This system of equations conserves charge. Charge can only flow between the electrodes and between the surface and bulk and between each other. The equations for the equilibrium potentials are given in equation (2), where $V_{U n, p}$ is the reference potential, $R$ is the ideal gas constant, $n$ is the number of transferred electrons, $F$ is Faraday's constant, $x_{n, p}$ is the positive and negative filling fraction (respectively), and $A_{n, p}$ are the fitting coefficients.

$$
\mathrm{V}_{\mathrm{Un}, \mathrm{p}}=\mathrm{V}_{\mathrm{U} 0 \mathrm{n}, \mathrm{p}}+\frac{\mathrm{RT}}{\mathrm{nF}} \log \left(\frac{1-\mathrm{x}_{\mathrm{n}, \mathrm{p}}}{\mathrm{x}_{\mathrm{n}, \mathrm{p}}}\right)+\frac{1}{\mathrm{nF}} \sum_{\mathrm{i}=1}^{\mathrm{N}_{\mathrm{n}, \mathrm{p}}} \mathrm{A}_{\mathrm{n}, \mathrm{p}}\left(2 \mathrm{x}_{\mathrm{n}, \mathrm{p}}-1\right)^{\mathrm{i}}-\frac{2 \mathrm{x}_{\mathrm{n}, \mathrm{p}}(\mathrm{i}-1)\left(1-\mathrm{x}_{\mathrm{n}, \mathrm{p}}\right)}{\left(2 \mathrm{x}_{\mathrm{n}, \mathrm{p}}-1\right)^{1-\mathrm{i}}}
$$

Importantly, while the fitting coefficients are estimated using simulated annealing, they are constrained to obey the second law of thermodynamics. This constraint helps to prevent overfitting and increases the interpretability of the model. It is enforced within the simulated annealing routine, by not allowing the routine to output an OCV curve which is not monotonic. 
The equations for the surface and ohmic overpotentials are given in equations (3) and (4), respectively, where $J_{p, n}$ is the current density, $J_{p 0, n 0}$ is the exchange current density, $V$ is the overpotential, and $\tau$ is the time constant for each overpotential:

$$
\begin{gathered}
\frac{\mathrm{d} \mathrm{V}_{\eta \mathrm{p}, \mathrm{n}}}{\mathrm{dt}}=\frac{\frac{\mathrm{RT}}{\mathrm{F} \alpha} \operatorname{asinh}\left(\frac{\mathrm{J}_{\mathrm{p}, \mathrm{n}}}{2 \mathrm{~J}_{\mathrm{p} 0, \mathrm{n} 0}}\right)-\mathrm{V}_{\eta \mathrm{p}, \mathrm{n}}}{\tau_{\eta \mathrm{p}, \mathrm{n}}} \\
\frac{\mathrm{dV} \text { ohm }}{\mathrm{dt}}=\frac{\mathrm{IR}-\mathrm{V}_{\mathrm{ohm}}}{\tau_{\mathrm{ohm}}}
\end{gathered}
$$

The equation for the thermal model is given in equation (5), where $I$ is the current, $R$ is the resistance, $\frac{\partial U}{\partial T}$ is the entropic coefficient, $h$ is the conductive coefficient, $A$ is the surface area (because the surface area of all cells is the same, we model $h A$ as a lumped parameter), $m$ is the cell mass, and $c_{p}$ is the specific heat.

$$
\frac{\mathrm{dT}}{\mathrm{dt}}=\frac{\mathrm{I}^{2} \mathrm{R}+\frac{\mathrm{I} \frac{\partial \mathrm{U}}{\mathrm{\partial T}}}{\mathrm{nF}}-\mathrm{hA}\left(\mathrm{T}-\mathrm{T}_{\mathrm{amb}}\right)}{\mathrm{mc}_{\mathrm{p}}}
$$

The thermal model is slightly specific to this dataset; any cooling model can be used in lieu of newtonian convection to best approximate cooling for any situation.

Parameter Estimation and Identification Different parameters were estimated using different techniques. Performance parameters, including all Redlich-Kister coefficients, the diffusion coefficients, the reference potentials, all thermal parameters, and time constants were estimated using simulated annealing. A stochastic optimization technique was chosen to avoid the parameter iden- 
tifiability issues described previously. The simulated annealing algorithm begins by evaluating the loss function at an initial point $\theta$. Then, using gibbs sampling, a random parameter is chosen to iterate, and a new parameter vector $\theta_{\text {candidate }}$ is created by adding a step $\lambda \mathcal{N}(0,1)$ where $\lambda$ depends on the magnitude of the selected parameter, and the loss function is then evaluated at $\theta_{\text {candidate }}$. If the new loss function is lower than the previous loss function, then $\theta$ is replaced by $\theta_{\text {candidate }}$. If the new loss function is higher than the previous loss function, then $\theta$ is replaced by $\theta_{\text {candidate }}$ with a probability calculated by the Boltzmann distribution:

$$
\mathrm{P}_{\text {acceptance }}=\mathrm{e}^{\frac{\left.\mathrm{L}\left(\theta_{\text {candidate }}\right)-\mathrm{L}(\theta)\right)}{\mathrm{T}}}
$$

Over the course of the optimization, the temperature $T$ is lowered to reduce the likelihood of moving to a less optimal parameter vector. This enables the algorithm to exploit and explore the parameter space. Exploration occurs when the temperature is relatively high, because the algorithm will allow movement to a higher loss value. This allows the algorithm to escape some local minima. Exploitation occurs when the temperature is relatively low, forcing the algorithm to move towards the local minima. All degradation parameters, including both mechanistic and UBDM methods, were estimated using simulated annealing.

We identified the aging parameters (charge and resistance) using a grid-based method. We chose this method for two reasons. Firstly, because there were only two parameters, and because of the aforementioned speed of the calculation of the loss function for this problem, a grid based method was tractable. Secondly, there was relatively little tolerance for optimization noise in these 
parameters, and the grid based method produced much less noise than a stochastic method such as simulated annealing. In the grid based method, upper and lower limits for charge and resistance were first identified based on physical constants. The lower resistance limit was defined as 0.01 ohms, and the upper resistance limit was defined as 0.05 ohms. The upper charge limit was defined as 26000 coulombs, and the lower charge limit was set to around 15000 coulombs. Using these limits, a 10 by 10 grid was created and the loss function was evaluated at each point on the grid. At the best points (those with the lowest loss function) the grid was further subdivided and the loss function was re-evaluated for a specified number of subdivisions and number of subgrid points.

As briefly mentioned in the article, we used a custom loss function to identify the parameters. That function given in equation (7), where a tilde over the variable indicates that it is the average experimental of the data, and a $\mathrm{t}$ subscript indicates that it is taken at timestep $\mathrm{t}, W$ indicates the weight given to each part, and the subscript of the $W$ 's indicates the part of the loss function to which that weight is applied. In this work, the weight was 10 for the voltage error and 1 for the other error terms.

$$
\mathrm{L}=\sum_{\mathrm{t}}\left(\mathrm{W}_{\mathrm{V}} \frac{\left|\mathrm{V}_{\mathrm{t}}-\tilde{\mathrm{V}}_{\mathrm{t}}\right|}{\overline{\mathrm{V}}}+\mathrm{W}_{\mathrm{T}} \frac{\left(\mathrm{T}_{\mathrm{t}}-\tilde{\mathrm{T}}_{\mathrm{t}}\right)^{2}}{\overline{\mathrm{T}}}\right)+\mathrm{W}_{\mathrm{MT}} \frac{\mid \max \left(\mathrm{T}_{\mathrm{t}}\right)-\max \left(\tilde{\mathrm{T}}_{\mathrm{t}} \mid\right)}{\overline{\mathrm{T}}}
$$

Degradation Modeling To model battery degradation, we follow a multistep process with three steps: first, find the parameters which change with aging. Then, fit those parameters to each (SOC) cycle for any given battery life-cycle, and identify whether or not the loss function is

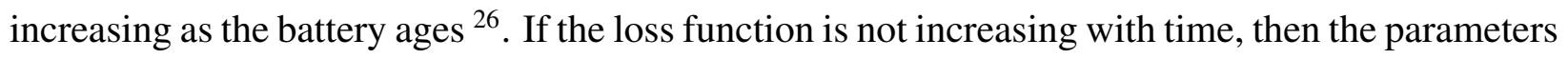


identified are the correct set of aging parameters. If the loss function is increasing with time, then the selection of the aging parameters needs to be revisited. As mentioned previously, because of the requirement for relatively low amounts of noise and the relatively low computational cost of estimating these parameters, the aging parameters were estimated using a grid-based method ${ }^{33}$.

Using this methodology, the precise degradation model can be chosen to fit the generated data once the parameters for each cycle have been estimated. For this work, we employed two separate degradation models. The first we refer to as the mechanistic model. It is based on physical principles and consists of charge loss due to SEI formation, Equation $8 \mathrm{a}$, active material isolation, Equation $8 \mathrm{cc}$, and lithium plating, Equation $8 \mathrm{~b} \mathrm{~b}^{27 / 28}$. Additionally, we add a resistance increase term, Eq. $8 \mathrm{~d})^{26}$.

$$
\begin{gathered}
\frac{\mathrm{dQ}_{\text {sei }}}{\mathrm{dt}}=\frac{\mathrm{K}_{\mathrm{SEI}} \mathrm{e}^{\frac{-\mathrm{E}_{\mathrm{SEI}}}{\mathrm{RT}}}}{2(1+\lambda \theta) \sqrt{\mathrm{t}}} \\
\mathrm{j}_{\mathrm{pl}}=\mathrm{i}_{0 \mathrm{pl}} \mathrm{e}^{-\frac{0.5 \mathrm{~F}}{\mathrm{RT}}\left(\mathrm{V}_{\mathrm{Un}}-\mathrm{V}_{\eta \mathrm{n}}\right)} \\
\frac{\mathrm{dQ}_{\mathrm{am}}}{\mathrm{dt}}=\mathrm{K}_{\mathrm{AM}} \mathrm{e}^{-\frac{\mathrm{E}_{\mathrm{AM}}}{\mathrm{RT}}} \mathrm{SOCI}_{\mathrm{app}} \\
\frac{\mathrm{dR}}{\mathrm{dt}}=\mathrm{w}_{\mathrm{d}}\left|\mathrm{I}_{\mathrm{app}}\right|
\end{gathered}
$$

We additionally have proposed a new degradation model. This model uses Universal Ordinary differential Equations (U-ODE) to estimate charge loss and resistance change. Neural Ordinary differential equations (NODE) are a model where the derivative of a function is approximated using a neural network ${ }^{29}$. U-ODE's are an extention of NODE's where only part of the time derivative is approximated using a neural network ${ }^{30}$. In this case, we have supplemented our mechanistic degradation model with a U-ODE to improve accuracy and generalization of predictions. The U- 
ODE neural network is a function of all of the other state variables of the system. Therefore, the degradation model is as such:

$$
\begin{aligned}
& \frac{\mathrm{dQ}_{\text {nonmechanistic }}}{\mathrm{dt}}=\mathrm{NN}(\mathrm{u}, \theta) \\
& \frac{\mathrm{dR}_{\text {nonmechanisitic }}}{\mathrm{dt}}=\mathrm{NN}(\mathrm{u}, \theta)
\end{aligned}
$$

where $u$ is defined as

$$
\mathrm{u}=\left[\mathrm{q}_{\mathrm{sp}}, \mathrm{q}_{\mathrm{bp}}, \mathrm{q}_{\mathrm{sn}}, \mathrm{q}_{\mathrm{bn}}, \mathrm{V} 0, \mathrm{~V}_{\eta \mathrm{n}}, \mathrm{V}_{\eta \mathrm{p}}, \mathrm{T}, \mathrm{R}, \mathrm{q}_{\max }\right]^{\mathrm{T}}
$$

and $\theta$ are the parameters of the neural network. We combine the mechanistic and non-mechanistic parts of the model to arrive at our final degradation model, the UBDM, which is written as:

$$
\begin{gathered}
\frac{\mathrm{dQ}}{\mathrm{dt}}=\frac{\mathrm{dQ}}{\mathrm{dt}}+\frac{\mathrm{dQ}_{\mathrm{AM}}}{\mathrm{dt}}+\mathrm{j}_{\mathrm{pl}}+\frac{\mathrm{dQ}_{\text {nonmechanistic }}}{\mathrm{dt}} \\
\frac{\mathrm{dR}}{\mathrm{dt}}=\frac{\mathrm{dR}_{\text {mechanistic }}}{\mathrm{dt}}+\frac{\mathrm{dR}_{\text {nonmechanistic }}}{\mathrm{dt}}
\end{gathered}
$$

The loss of charge in the degradation model always occurs at the anode surface.

\section{References}

1. Schmuch, R., Wagner, R., Hörpel, G., Placke, T. \& Winter, M. Performance and cost of materials for lithium-based rechargeable automotive batteries. Nat. Energy 3, 267-278 (2018). URLhttps://doi.org/10.1038/s41560-018-0107-2. 
2. Harlow, J. E. et al. A wide range of testing results on an excellent lithium-ion cell chemistry to be used as benchmarks for new battery technologies. J. Electrochem. Soc. 166, A3031-A3044 (2019). URL https://doi.org/10.1149/2.0981913 jes.

3. Tomaszewska, A. et al. Lithium-ion battery fast charging: A review. eTransportation 1, 100011 (2019). URLhttps://doi.org/10.1016/j.etran.2019.100011.

4. Arnot, M. Is a 63-Year-Old Seaplane With an Electric Engine the Future of Air Travel? . https://www.nytimes.com/2019/12/26/travel/electric-air-travel. html (2019). Accessed Date: 2-July-2020.

5. Etherington, D. Watch a full test flight of Lilium's all-electric urban aircraft . https://techcrunch.com/2019/12/18/ watch-a-full-test-flight-of-liliums-all-electric-urban-aircraft/ (2019). Accessed Date: 2-July-2020.

6. Duffy, M. J., Wakayama, S. R. \& Hupp, R. A study in reducing the cost of vertical flight with electric propulsion. In 17th AIAA Aviation Technology, Integration, and Operations Conference (American Institute of Aeronautics and Astronautics, 2017). URL https://doi.org/10.2514/6.2017-3442,

7. Vertipedia. V/stol aircraft and propulsion concepts. https://vertipedia-legacy. vtol.org/vstol/wheel.htm(2019). Accessed Date: 2-July-2020. 
8. Kim, H. D., Perry, A. T. \& Ansell, P. J. A review of distributed electric propulsion concepts for air vehicle technology. In 2018 AIAA/IEEE Electric Aircraft Technologies Symposium (EATS), $1-21(2018)$.

9. Lillian Gipson, ed. NASA Embraces Urban Air Mobility, Calls for Market Study. https:// WwW.nasa.gov/aero/nasa-embraces-urban-air-mobility (2017). Accessed Date: 2-July-2020.

10. Langford, J. S. \& Hall, D. K. Electrified aircraft propulsion. The Bridge 50, 21-27 (2020).

11. Fredericks, W. L., Sripad, S., Bower, G. C. \& Viswanathan, V. Performance metrics required of next-generation batteries to electrify vertical takeoff and landing (vtol) aircraft. ACS Energy Lett. 3, 2989-2994 (2018).

12. Schäfer, A. W. et al. Technological, economic and environmental prospects of allelectric aircraft. Nat. Energy 4, 160-166 (2018). URL https://doi.org/10.1038/ S41560-018-0294-x.

13. Sridharan, A., Govindarajan, B. \& Chopra, I. A scalability study of the multirotor biplane tailsitter using conceptual sizing. J. Am. Helicopter Soc. 65, 1-18 (2020).

14. German, B., Daskilewicz, M., Hamilton, T. K. \& Warren, M. M. Cargo delivery in by passenger eVTOL aircraft: A case study in the san francisco bay area. In 2018 AIAA Aerospace Sciences Meeting (American Institute of Aeronautics and Astronautics, 2018). URL https://doi.org/10.2514/6.2018-2006 
15. Reniers, J. M., Mulder, G. \& Howey, D. A. Review and performance comparison of mechanical-chemical degradation models for lithium-ion batteries. J. Electrochem. Soc. 166, A3189-A3200 (2019). URL https: / / doi.org/10.1149/2.0281914 jes.

16. Ng, M.-F., Zhao, J., Yan, Q., Conduit, G. J. \& Seh, Z. W. Predicting the state of charge and health of batteries using data-driven machine learning. Nat. Mach. Intell. 2, 161-170 (2020). URL https://doi.org/10.1038/s42256-020-0156-7.

17. Severson, K. A. et al. Data-driven prediction of battery cycle life before capacity degradation. Nat. Energy 4, 383-391 (2019). URL https://doi.org/10.1038/ s41560-019-0356-8.

18. Zuo, Y. et al. Performance and cost assessment of machine learning interatomic potentials. J. Phys. Chem. A 124, 731-745 (2020). URL https://doi .org/10.1021/acs . jpca. $9 \mathrm{~b} 08723$.

19. Raissi, M., Yazdani, A. \& Karniadakis, G. E. Hidden fluid mechanics: Learning velocity and pressure fields from flow visualizations. Science 367, 1026-1030 (2020). URL https: //doi.org/10.1126/science.aaw4741.

20. Bezanson, J., Karpinski, S., Shah, V. B. \& Edelman, A. Julia: A fast dynamic language for technical computing. arXiv preprint arXiv:1209.5145 (2012).

21. Saha, B. \& Goebel, K. Battery data set. NASA AMES prognostics data repository (2007).

22. Howey, D. A. Tools for battery health diagnostics and prediction. Electrochem Soc Interface 28, 55-56 (2019). URL https://doi.org/10.1149/2.f06191if. 
23. Bole, B., Kulkarni, C. S. \& Daigle, M. Adaptation of an electrochemistry-based li-ion battery model to account for deterioration observed under randomized use. Tech. Rep., SGT, Inc. Moffett Field United States (2014).

24. Forman, J. C., Moura, S. J., Stein, J. L. \& Fathy, H. K. Genetic identification and fisher identifiability analysis of the doyle-fuller-newman model from experimental cycling of a LiFePO4 cell. J. Power Sources 210, 263-275 (2012). URL https://doi.org/10.1016/j. jpowsour.2012.03.009

25. Kirkpatrick, S., Gelatt, C. D. \& Vecchi, M. P. Optimization by simulated annealing. Science 220, 671-680 (1983). URL https://science.sciencemag.org/content/ 220/4598/671. https://science.sciencemag.org/content/220/4598/ 671.full.pdf.

26. Daigle, M. \& Kulkarni, C. S. End-of-discharge and end-of-life prediction in lithium-ion batteries with electrochemistry-based aging models. In AIAA Infotech @ Aerospace (American Institute of Aeronautics and Astronautics, 2016). URL https://doi .org/10.2514/ $6.2016-2132$

27. Jin, X. et al. Physically-based reduced-order capacity loss model for graphite anodes in li-ion battery cells. J. Power Sources 342, 750 - 761 (2017). URL http://www. sciencedirect.com/science/article/pii/s037877531631802X.

28. Yang, X.-G., Leng, Y., Zhang, G., Ge, S. \& Wang, C.-Y. Modeling of lithium plating induced aging of lithium-ion batteries: Transition from linear to nonlinear aging. J. Power Sources 
360, 28 - 40 (2017). URL http://www.sciencedirect.com/science/article/ pii/S0378775317307619.

29. Chen, R. T., Rubanova, Y., Bettencourt, J. \& Duvenaud, D. K. Neural ordinary differential equations. In Advances in neural information processing systems, 6571-6583 (2018).

30. Rackauckas, C. et al. Universal differential equations for scientific machine learning. arXiv preprint arXiv:2001.04385 (2020).

31. Daigle, M. J. \& Kulkarni, C. S. Electrochemistry-based battery modeling for prognostics. In Annual Conference of the Prognostics and Health Management Society, vol. 4 (2013).

32. Karthikeyan, D. K., Sikha, G. \& White, R. E. Thermodynamic model development for lithium intercalation electrodes. J. Power Sources 185, 1398-1407 (2008). URL https://doi. org/10.1016/j.jpowsour.2008.07.077

33. Kurchin, R., Romano, G. \& Buonassisi, T. Bayesim: A tool for adaptive grid model fitting with bayesian inference. Comput. Phys. Commun. 239, 161 - 165 (2019). URL http: //www.sciencedirect.com/science/article/pii/s0010465519300414.

Acknowledgements This work was supported by Airbus $\mathrm{A}^{\wedge} 3$. The authors would also like to thank Ananth Sridharan for his inputs on the relevance of battery SOC and SOH estimation for electric aircraft sizing, Romain Teulier for coordinating the battery testing presented in the article. E. F. and D. C. performed the work reported in this paper during their time as employees at Airbus $\mathrm{A}^{\wedge} 3$.

Contributions A.B. and L.F. were the main developers of the software code for cellFit. S.S., A.B., L.F. 
and V.V. developed the underlying modeling approach. M.G. and V.V. developed the simulated annealing optimization approach. E.F. and D.C. coordinated the generation of the experimental dataset. A.B., S.S., E.F. and V.V. wrote the paper. All authors read the paper and provided input.

Competing Interests The authors declare that they have no competing financial interests.

Correspondence Correspondence and requests for materials should be addressed to V. Viswanathan (email: venkvis@cmu.edu). 